\title{
Success rate of first haemodialysis post tunnelled dialysis catheter insertion
}

\author{
Dahwa $\mathbf{R}^{1}$, Martinez-Garcia J ${ }^{1}$, Lee $\mathbf{C}^{1}$ and Ratanjee $\mathrm{SK}^{* 1,2}$ \\ ${ }^{1}$ Renal Department, Royal Brisbane and Women's Hospital, Australia \\ ${ }^{2}$ School of Medicine, University of Queensland, Australia
}

\section{Background}

Vascular access has been described as the Achilles heel of haemodialysis(HD) [1]. The arteriovenous fistula (AVF) is still the preferred vascular access for haemodialysis especially in the era of the Fistula First Initiative [2]. However due to a number of factors, including the presence of co-morbidities like diabetes and peripheral vascular disease, lack of vascular access due to late nephrological referral [3], and more patients of advanced age on HD some patients still start dialysis with a tunnelled dialysis catheter (TDC). Also, patients may need a catheter if there are native access related complications. Peritoneal dialysis (PD) patients may require haemodialysis if there are temporary problems with undertaking PD.

In Australia a significant proportion of patients still commence renal replacement therapy (RRT) with a TDC. The percentage of patients with a TDC at the time of first RRT has remained stable with values ranging from 37-39\% from 2006-2009 [4].

In the Metro North area of Queensland, Australia, The Royal Brisbane and Women's Hospital (RBWH) radiology service is utilised by several regional centres for TDC insertion. Patients are usually kept in hospital to ensure that their first dialysis post catheter insertion is successful. Only then do patients return to their original dialysis unit. This leads to an increased length of stay and demand on the dialysis unit at our institution. In view of resource implications, we set out to determine if this strategy was essential.

\section{Study aim}

The aim of this study was to prospectively evaluate the success rate of the first dialysis session post TDC insertion for catheters inserted over an 8 month period.

\section{Methods}

A prospective analysis of catheter insertions performed over an 8 month period was done. The outcomes of the first dialysis session post catheter insertion were documented by two designated nursing staff.

All TDCs were inserted by a radiologist in the radiology department with ultrasound guidance and fluoroscopy to ascertain appropriate catheter positioning in the right atrium. All TDCs were inserted in the right (preferably) or left internal jugular vein.

For the purposes of this study a blood flow rate (BFR) of $250 \mathrm{~mL} /$ minute was considered to be sufficient as the patient dialysed for 5 hours. Patients new to dialysis dialysed for 2-3 hours only on their first dialysis and a blood flow of $200 \mathrm{ml} / \mathrm{min}$ was deemed sufficient.

A BFR of at least $250 \mathrm{ml} / \mathrm{min}$ in an average adult $(60-70 \mathrm{~kg})$ offers adequate dialysis in a dialysis time of approximately 4 hours dialysis [5]. Subsequent BFRs were increased as required by the referring unit.

\section{Results}

In the 8 month period a total of 90 catheters were inserted in 74 patients. One patient who had acute kidney injury recovered and did not require dialysis despite having a TDC inserted and was excluded from the study analysis. Of the remaining 89 TDCs, 88 were Hemostar $^{\mathrm{TM}}$ (Bard Access Systems Incorporated, USA) catheters and 1 was a Palindrome ${ }^{\mathrm{TM}}$ catheter. Our data analysis is of the 88 Hemostar catheters.

We further analysed whether insertion site (left or right internal jugular) had a bearing on the success rate. We also sought to see whether there was a difference in patients who were having their first catheter and patients having subsequent catheters.

Of the 88 procedures $59(67 \%)$ had a TDC for the first time and twenty-nine $(33 \%)$ had at least one previous catheter. The majority of the catheters were inserted in the right internal jugular (90\% and $72 \%)$ respectively in the two groups.

Only two patients failed to achieve a BFR of $250 \mathrm{ml} / \mathrm{min}$ during the first dialysis session after catheter insertion. One of the patients had her first catheter inserted into the right internal jugular vein and the other patient had her second catheter into the left internal jugular vein with both tips in the right atrium. This gives an overall success rate of $98 \%$.

\section{Discussion}

Early catheter dysfunction is usually related to the insertion process. For the purposes of this study we used the blood flow rate to define dysfunction but dysfunction can also be identified by high resistance pressures whilst on dialysis as well as recirculation of dialysed blood [6].

Ideally the catheter should be placed in the right internal jugular vein and the tip placed within the atrium. Placement in the right atrium enables high blood flow rates to be achieved and the right internal jugular offers the most direct access to the atrium. It has to be noted however that in obese individuals and large breasted females that the

Correspondence to: SK Ratanjee, Renal Department, Royal Brisbane and Women's Hospital, Australia; School of Medicine, University of Queensland, Australia, E-mail: dockidney@gmail.com

Received: March 11, 2017; Accepted: March 23, 2017; Published: March 27, 2017 
tip of the catheter moves up when they change from a supine to an upright position and thus the operator has to take cognisance of this at the time of insertion [7]. Catheter tip migration also occurs more commonly with catheters that are placed within the subclavian vessels. The position of the catheters in our study was checked by fluoroscopy thus limiting occurrence of malposition and kinks.

Analysis of our results showed that a significant proportion of the catheters were accessed through the right internal jugular vein and only $18 \%$ had the left internal jugular vein accessed. Accessing the subclavian vein is associated with a higher risk of central vein stenosis and thus is generally avoided.

The rate of unsuccessful catheter insertions was low in this study (Table 1). Thus patients who come from outside of our hospital district need not wait to have their first dialysis at our centre prior to returning to their usual dialysis unit. This reduces the duration of hospital stays and also reduces pressure on the dialysis unit at our institution.
Catheter dysfunction however is a significant problem often requiring another catheter. This group accounted for $20 \%$ of all the catheter insertions (Figures 1 and 2). This shows that even if the initial $\mathrm{HD}$ is successful ongoing surveillance as recommended by the KDOQI clinical practice guidelines is required, particularly for those who have a catheter as long term vascular access.

\section{Conclusions}

This is the first study to our knowledge to evaluate dialysis BFRs for the first dialysis session after insertion of Hemostar TDC. With the use of techniques such as ultrasound and fluoroscopy the success rate of catheters is exceptionally high. Patients coming from regional centres need not have their first dialysis session at our institution to ascertain adequate flows prior to returning to their usual dialysis unit.

Catheter dysfunction is a significant problem and even if initial dialysis BFRs are sufficient ongoing surveillance is required.

Table 1. Patient demographics.

\begin{tabular}{|c|c|}
\hline Male to Female ratio & $2: 1$ \\
\hline Mean Age & $55 y e a r s$ \\
\hline Out of district & $20(22 \%)$ \\
\hline Number of patients with repeated catheter insertions & $11(12.5 \%)$ \\
\hline Successful first haemodialysis & $86(98 \%)$ \\
\hline
\end{tabular}

$\square \mathrm{AKI} \quad \mathrm{AKI} / \mathrm{CKD}$

CKD V symptomatic failed PD

poor flows

Peritonitis thrombosed AVF

failed transplant catheter dislodged

infected permacath

PD dysfucntion

Infected AVG

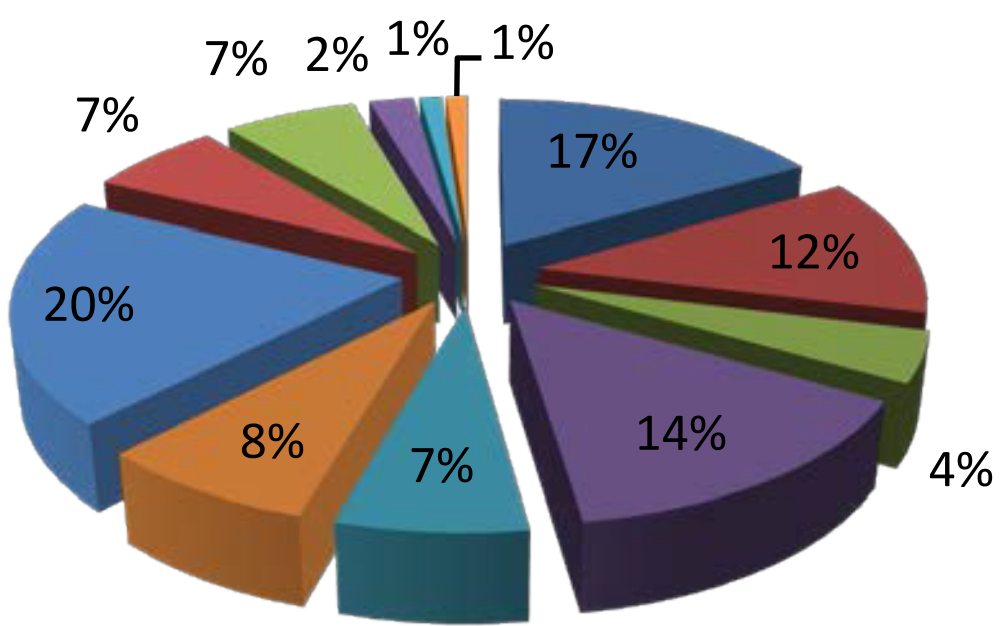

AKI: Acute Kidney Injury 17\%

AKI/CKD: Acute Kidney Injury on Chronic Kidney Injury 12\%

CKD V symptomatic: Symptomatic Stage V Chronic Kidney Disease 14\%

Thrombosed AVF: Thrombosed Arteriovenous fistula 7\%

Failed PD: Failed Peritoneal Dialysis 7\%

PD dysfunction: Peritoneal Dialysis dysfunction (The group PD Dysfunction included patients who had tenckoff catheter malposition and patients who had had abdominal surgery and were resting their peritoneum.) $7 \%$

Infected AVG: Infected Arteriovenous graft 1\% 


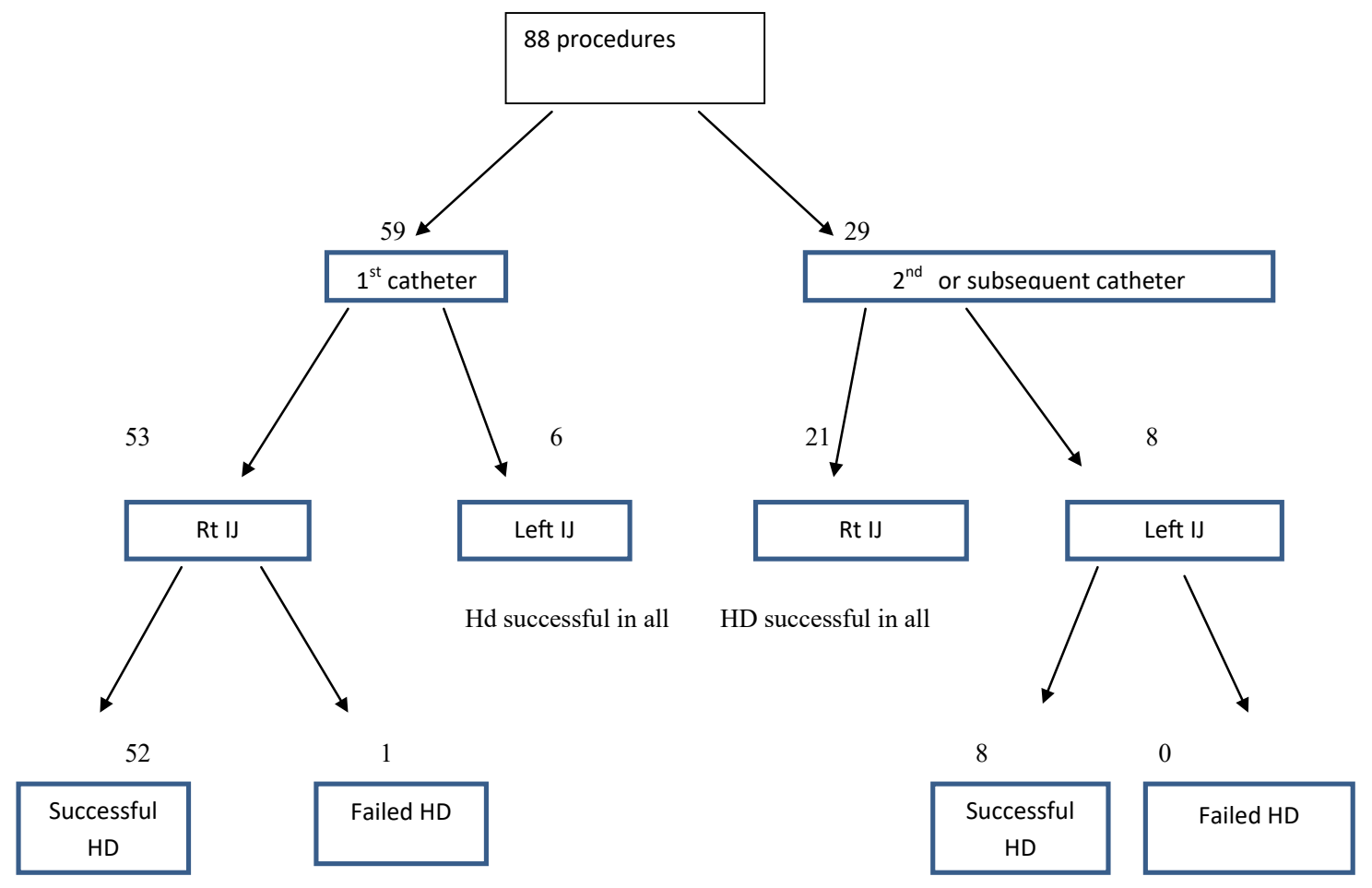

Rt IJ: Right internal jugular

Left IJ: Left internal jugular

HD: haemodialysis

Figure 2. Breakdown of catheter number, insertion site and HD outcome.

\section{References}

1. Winkelmayer WC (2011) Tackling the Achilles' heel of hemodialysis. $N$ Engl J Med 364: 372-374.[Crossref]

2. Vascular Access Work Group (2006) Clinical practice guidelines for vascular access. Am J Kidney Dis 48: S248-273. [Crossref]

3. Knuttinen MG, Bobra S, Hardman J, Gaba RC, Bui JT, Owens CA (2009) A review of evolving dialysis catheter technologies. Semin Intervent Radiol 26: 106-114. [Crossref]
4. ANZDATA (2010) The 33rd Annual Report 2010 Report.

5. Mandolfo S, Piazza W, Galli F (2002) Central venous catheter and the hemodialysis patient: a difficult symbiosis. J Vasc Access 3: 64-73. [Crossref]

6. Leblanc M, Bosc JY, Paganini EP, Canaud B (1997) Central venous dialysis catheter dysfunction. Adv Ren Replace Ther 4:377-389. [Crossref]

7. Schon D, Whittman D (2003) Managing the complications of long-term tunneled dialysis catheters. Semin Dial 16: 314-322. [Crossref]

Copyright: (2017 Dahwa R. This is an open-access article distributed under the terms of the Creative Commons Attribution License, which permits unrestricted use, distribution, and reproduction in any medium, provided the original author and source are credited. 